\title{
Cross-talk between neurons and glia: highlights on soluble factors
}

F.C.A. Gomes,

T.C.L.S. Spohr, R. Martinez and V. Moura Neto
Instituto de Ciências Biomédicas, Departamento de Anatomia,

Universidade Federal do Rio de Janeiro, Rio de Janeiro, RJ, Brasil

\section{Correspondence}

F.C.A. Gomes

Departamento de Anatomia

ICB, UFRJ, CCS, Bloco F

21949-590 Rio de Janeiro, RJ

Brasil

Fax: + 55-21-562-6493

E-mail: fgomes@ anato.ufrj.br

Presented at

SIMEC 2000 - International

Symposium on Extracellular

Matrix, Angra dos Reis, RJ,

Brazil, September 24-27, 2000

Research supported by PRO NEX-MCT (No. 052/97), CAPES-CO FECUB,

FAPERJ, FINEP, and CEPG-UFRJ.

Received October 31, 2000 Accepted February 20, 2001

\section{Abstract}

The development of the nervous system is guided by a balanced action between intrinsic factors represented by the genetic program and epigenetic factors characterized by cell-cell interactions which neural cells might perform throughout nervous system morphogenesis. Highly relevant among them are neuron-glia interactions. Several soluble factors secreted by either glial or neuronal cells have been implicated in the mutual influence these cells exert on each other. In this review, we will focus our attention on recent advances in the understanding of the role of glial and neuronal trophic factors in nervous system development. We will argue that the functional architecture of the brain depends on an intimate neuron-glia partnership.

\section{Introduction}

A central objective of developmental biology is to elucidate the mechanisms that specify particular cell types during animal development. The central nervous system (CNS) provides an interesting model to identify the development programs that create cell type-specific patterns. Within this context, cell-cell interactions are of fundamental importance in the organization and maintenance of the nervous system architecture. For a long time glial cells were regarded as somewhat passive companions to neurons that performed a variety of essential but almost perfunctory duties. Today, however, more than a century after their description by Virchow, increasing evidence has been accumulating indicating that neurons and glial cells have an intimate and plastic morphological and functional relationship.

\section{Key words}

- Neuron-glia interaction

- Trophic factors

- Astrocyte

- Neuron
- Nervous system 


\section{Soluble factors as neuron-glia interaction mediators}

\section{Neuron-glia interactions: implications in the matching of axons/oligodendrocytes and Schwann cell number}

Most of our knowledge concerning neuron-glia interactions concerns the effects of glial cells on neuronal morphogenesis. However, evidence has accumulated in the past years pointing to a mutual influence between these two cell types.

Most data about the action of neuronal factors on glial cells concern neuron-oligodendrocyte interactions. Nearly ten years ago, glia-promoting factors (GPF), brain peptides which stimulate growth of specific macroglial populations in vitro, were identified (9). First discovered in the goldfish visual system, peptides with similar properties were later identified in the mammalian brain. Some of them, GPF1 and GPF3, are secreted by neurons and represent a source of oligodendroglia-stimulating factors (9). At present, it is widely recognized that the survival and proliferation of oligodendrocytes are highly dependent on neuronal contact and neuronal soluble factors (2). Soluble factors secreted by axons apparently control oligodendrogenesis by stimulating oligodendrocyte precursor proliferation. Once cell division has ceased the cells must meet a nonmyelinized axon in order to survive. Such refinement of oligodendrocyte-neuron interaction plays a crucial role in matching the number of oligodendrocytes and myelinized axons, thus ensuring that the number of free axons does not exceed that of oligodendrocytes. Transection of the optic nerve during development results in a severe reduction in the number of oligodendrocytes, suggesting a dependence of developing oligodendroglia on neuronal survival factors (10). Furthermore, transection of the adult optic nerve results in a substantial reduction in the expression of the myelin-related gene by oligodendrocytes
(11). Knockout animals for the $b c l 2$ gene, a modulator of apoptosis, in addition to the death of a large amount of retina ganglion cells (RGC), present increased oligodendrocyte death, emphasizing the role of neuronal factors in sustaining oligodendrocyte survival and/or proliferation (2). Additionally, neurons and neuronal extracts are known to provide a mitogenic signal for both immature and mature oligodendrocytes $(12,13)$.

\section{The role of neuregulin}

Several candidate mitogens and survival factors have been identified that are effective at different stages in the oligodendrocyte lineage: platelet-derived growth factor (PDGF) (14), basic fibroblast growth factor (bFGF), neurotrophin-3 and insulin growth factor (2). All of these factors are released by astrocytes and bFGF and PDGF are expressed and released by neurons as well. Recently, members of the bone morphogenetic protein family have been shown to inhibit oligodendrocyte differentiation in vitro (15). The most promising candidate for the neuronal control of oligodendrocyte progenitor proliferation is glial growth factor 2 (GGF2) from the neuregulin family (16). The NDF/neuregulin family includes more than a dozen growth and differentiation factors that share an epidermal growth factor (EGF)-like motif, serving as the receptor-binding domain. GGF2 has been reported as a potent mitogen and survival factor for the oligodendrocyte lineage and its progenitors and as an inhibitor of pro-oligodendrocyte differentiation. Several studies have shown that oligodendrocyte proliferation is under the control of several mitogens that operate at different stages in the oligodendrocyte lineage. While GGF and PDGF are likely to regulate early progenitors, GGF and FGF may function at later times on more differentiated cells (16).

A similar neuronal control of cell proliferation and differentiation has been reported for Schwann cells, involved in peripheral 
nervous system myelinization (17). GGF is a strong mitogenic molecule for Schwann cells, secreted by neural precursors of peripheral ganglia $(17,18)$. Members of the neuregulin family reduce the apoptosis of mature Schwann cells upon axotomy (15), probably reflecting a role in attaining the appropriate ratio of neurons to Schwann cells in adults (19). The severe deficiency of Schwann cells in mice in which the neuregulin-1 gene has been inactivated provides striking support for the key role of this growth factor in Schwann cell development (20). Furthermore, GGF2 is also involved in glial fate determination. This factor causes neural crest stem cells to acquire a glial phenotype instead of a neuronal fate (21). Indeed, GGF2 mutants lack Schwann cell precursors along peripheral projections from the spinal cord (20).

Although axons are required for oligodendrocyte differentiation and myelination, tissue culture studies suggest that initially they promote proliferation and delay myelination of oligodendrocyte progenitors, possibly by secreting GGF (for a review, see 2). Similar effects on Schwann cell myelination have been reported, indicating that high levels of neuronal mitogens can promote proliferation and arrest myelination in both the CNS and the peripheral nervous system. By promoting the survival and proliferation of both immature oligodendrocytes and Schwann cells, neuregulins may therefore play a crucial role during development in adjusting the final number of myelinating glial cells to the number of available axons.

\section{The role of neurotransmitters}

In addition to traditional growth factors, recognition of the role of nonconventional trophic factors such as neurotransmitters has been growing for the last ten years. Most of them might regulate neurogenesis as well as gliogenesis (22). Gallo et al. (23) have demonstrated by using purified cortical oligo- dendrocyte precursor cells grown in primary culture that non-NMDA glutamate receptor agonists inhibit cell proliferation. Recently, these investigators showed that in cerebellar slice cultures glutamate acts as an antimitotic signal at all proliferative stages in the oligodendrocyte lineage. Treatment of cerebellar slices with kainate or AMPA caused a 55 and $37 \%$ decrease, respectively, in the mRNA levels of the oligodendrocyte maturation marker CNPase (2'3' cyclic nucleotide 3' phosphodiesterase). In contrast, treatment with the glutamate receptor antagonist significantly increased CNPase RNA levels by $32 \%(24)$.

In addition to PDGF, GGF, bFGF and others, glutamate and its receptors are likely to be part of the complex network of signals that regulate oligodendrocyte development in vivo. Understanding the cues provided by neuronal cells to oligodendrocytes and Schwann cells might open new perspectives in elucidating myelination and regeneration in the CNS as well as in the peripheral nervous system.

\section{Neuron-microglia interactions: the basis of nervous system inflammatory response}

Neuron-derived growth factors have been implicated in the modulation of nervous system inflammatory response. In the developing CNS, invading monocytes proliferate and are transformed into ameboid microglia, an atypical subclass of glial cells, also called brain macrophages (25). Proliferation of microglia can be stimulated by colony-stimulating factors (CSF), such as CSF-1, also called macrophage CSF and granulocyte macrophage CSF. Recently, Dobbertin et al. (26) demonstrated that rat neurons stimulate macrophage proliferation by increasing their mitogenic response to CSF. This effect was shown to be mediated by secretion of transforming growth factor $\beta 2$ (TGF- $\beta 2$ ), a member of the TGF- $\beta$ superfamily which is composed of a range of functional and structural 
factor subclasses with a range of cellular actions and developmental regulatory effects on organogenesis, pattern formation, modulation of extracellular matrix and terminal differentiation (27). All those data provided evidence that neurons might support microglia growth during development by secreting TGF- 32 , which stimulates the proliferation of brain macrophages and their precursors infiltrating CNS tissue.

Recently, a new loop in neuron-microglia interaction has emerged. Noda et al. (28) have identified functional subtypes of glutamate receptors in rat cerebral microglia. Although the physiological role of these receptors in microglia remains unclear the authors reported that their activation enhances tumor necrosis factor alpha (TNF- $\alpha$ ) production by microglia. Since TNF- $\alpha$ rapidly increases after excitotoxic, ischemic and traumatic brain injury, it is possible that elevated levels of glutamate at pathological sites may directly activate receptors on microglia, inducing a prompt response to injury. Another putative mediator of neuron-microglia signaling is platelet-activating factor (PAF) which is a potent phospholipid mediator that plays several roles in neuronal function and brain development. Aihara et al. (29) demonstrated that cultivated neurons synthesized PAF following stimulation with glutamic acid. Microglia, which express functional PAF receptors, showed a marked chemotactic response to this factor, pointing to PAF as a key messenger in neuron-microglia interactions.

\section{Neuron-astrocyte interactions}

\section{Role of astrocytes in neuronal development}

The normal development of the vertebrate nervous system entails the death of a great quantity of the neurons originally generated. This naturally occurring cell death is regulated by the availability of specific neurotrophic factors that promote neuronal sur- vival (30).

For a long time it has been suspected that astrocytic cells play a trophic role in supporting neurons (31). Factors of the FGF, TGF and EGF families play an important role in early neurogenesis $(32,33)$ and all of them are potentially secreted by astrocytes. Members of the EGF and FGF families are potent mitogens for multipotential neural progenitors and are highly implicated in several aspects of neurogenesis (22). Members of the TGF- $\beta$ family such as TGF- $\beta$ itself and glial cell line-derived neurotrophic factor have been reported to have a broad spectrum of action during nervous system development. Both are known to favor survival of dopaminergic neurons in vivo as well as in vitro $(34,35)$. We have recently reported that TNF- $\beta$ and EGF secreted by cultured cerebellar astrocytes in response to thyroid hormone $\left(\mathrm{T}_{3}\right)$ treatment can modulate neuronal proliferation (5). Cerebellar neurons maintained in the presence of conditioned medium derived from $\mathrm{T}_{3}$-treated astrocytes presented a three-fold increase in the incorporation of the proliferation marker, bromodeoxyuridyl. Neuronal survival was not affected by GGF, suggesting a prior function for TNF- $\beta$ and EGF in glia-mediated neuronal proliferation. Additionally, Trentin A, Alvarez M and Moura Neto V (unpublished results) identified several other growth factors secreted by astrocytes in response to $\mathrm{T}_{3}$ such as aFGF and bFGF. All of these factors might be potentially trophic for neurons and might constitute a bridge between thyroid hormone and neurogenesis (for a review, see 36).

During CNS development, neurons must extend projections in order to establish their connections. Growing axons navigate toward their targets in response to a variety of guidance signals in their surrounding environment. These cues include diffusible attractive or repellent molecules secreted by the intermediate or final cellular targets of the axons. Glial cells have been exhaustively 
reported as a source of asymmetric cues during axonal navigation (37). Commissural and decussation formation in the nervous system, such as the optic chiasm and the floor plate of the nervous system, is dependent on the interaction of growth axons and resident glia of these regions. Several adhesion and soluble molecules involved in such interactions have already been reported. Netrin-1, a laminin-related molecule containing three EGF repeat motifs, is one of the better known soluble signals. Netrin-1 is important for axon guidance to the midline of the brain. Netrin-deficient mice show additional defects in the corpus callosum as well as hippocampal and anterior commissures, and have been shown to attract ventrally decussating axons in the developing brainstem (38).

In agreement with in vivo studies, several in vitro lines of evidence have implicated astrocyte-soluble factors in neuronal morphogenesis. Neuronal polarity, which is crucial for neural circuits, is highly modulated by glial cells. Sympathetic neurons maintained in vitro in the presence of astrocytes extend axons and dendrites, while in the absence of astrocytes they extend solely axons. Such dendritic neuritogenesis is induced by bone morphogenetic proteins, a subclass of the TGF- $\beta$ superfamily involved in many aspects of neuronal maturation (39).

Additional evidence for the influence of astrocyte-soluble factors on neuronal morphogenesis has been provided by studies of Garcia-Abreu et al. $(6,40)$ who demonstrated that astrocytes derived from distinct regions of the midbrain can differently modulate neurite extension. Astrocytes derived from the lateral region of the mesencephalon are highly permissive to neurite outgrowth, whereas those derived from the midline proved to be restrictive to neuritogenesis (6). These astrocyte populations exhibited great heterogeneity in the content of soluble proteoglycans secreted in the medium (40). These discrepancies may account for the differences observed in their ability to support neurite outgrowth.

Taken together, the above data provide evidence that astrocyte-soluble factors play an important role in several steps of neuronal morphogenesis from the early events of neuronal precursor proliferation until later periods of neuronal differentiation and establishment of neural circuits.

\section{Role of neuronal factors in astrocyte morphogenesis}

While there is compelling evidence of the effects of astrocyte factors on neurons, their effects on astrocytes have not been determined. Some of our knowledge about astrocyte biology came from studies of the visual system. The vertebrate eye provides an interesting system to study cell-cell communication. During development, cells from several different sources come together in a coordinated fashion to form the final structure (41). The retina itself is composed of cells of different developmental origins, whose numbers must presumably be matched to one another by cell-cell interactions. Most of the cells of the neural retina are generated by multipotential neuroepithelial precursors that reside near the outer surface of the retina. In contrast, retinal astrocytes originate from the optic stalk and migrate across the inner surface of the retina. The migrating astrocytes form a glial network that spreads radially in close association with the RGC axons. Such invasion by astrocytes has been reported to be mediated by secretion of PDGF by RGC. This factor is expressed and secreted by RGC while PDGF receptor alpha (PDGFR $\alpha$ ), an isoform of the tyrosine kinase PDGFR, is expressed in retinal astrocytes. By inhibiting PDGF signaling with a neutralizing anti-PDGFR $\alpha$ or a soluble extracellular fragment of PDGFR $\alpha$, Fruttiger et al. (42) impaired astrocyte network development. Apparently, PDGF mediates a short paracrine interaction between RGC and as- 
trocytes during retina development.

Besides retinal astrocyte migration, their morphology is also strongly influenced by RGC axons. At the periphery of the cat retina, where RGC axons are sparse, astrocytes adopt a stellate shape in contrast to the strongly elongated form present in RGC-rich regions. Recently, Gargini et al. (43) provided evidence that the astrocyte axon-related morphology is induced by a signal derived from spikes. Although the nature of the astrocyte trophic signal has not been identified, a possibility considered by the authors is that it might be a released polypeptide acting through astrocyte receptors.

Although neuronal effect mechanisms on astrocytes are still far from being well understood, increasing evidence has been accumulated pointing at neurons as modulators of astrocyte gene expression and differentiation (44-47). A way to study astrocyte differentiation is by evaluating levels of proteins whose expression patterns vary during astrocyte development such as the intermediate filament glial fibrillary acidic protein (GFAP) $(46,47)$, the enzyme glutamine synthetase (48) and glutamate transporters (49), among others. Two main subtypes of glutamate transporters have been described in glia, i.e., GLAST, with expression predominating at early stages, and GLT-1 whose expression progressively increases with maturity. Swanson et al. (44) have reported that neurons can modulate astrocyte glutamate transporter expression in vitro. In the absence of neurons, cortical astrocytes maintain polygonal shapes and express only the GLAST transporter. When co-cultured with a neuronal layer, many of the astrocytes assume a stellate shape and express GLT-1. These findings support the general principle that normal expression of GLT-1 protein by astrocytes requires a neuronal signal, suggesting that neurons can modulate astrocyte differentiation. Although the nature of this neuronal signal remains to be identified, recent reports have clearly demonstrated that GLT-
1 and GLAST expression is modulated by neuronal soluble factors rather than by cell contact $(50,51)$.

By using transgenic mice bearing $2 \mathrm{~kb}$ of the 5' flanking region of the astrocyte maturation marker GFAP gene linked to the $\beta$ galactosidase reporter gene, we have demonstrated that cortical neurons can induce the GFAP gene promoter followed by transgenic astrocyte differentiation, by secreting brain region-specific soluble factors (47). This event was dependent on the brain regional origin of the neurons since cerebral hemisphere neuronal factors were unable to induce the GFAP gene promoter of midbrain and cerebellar astrocytes. Several growth factors are putative candidates in mediating such effect (46). Further analysis of the GFAP gene promoter would contribute to the identification of the neuronal-derived astrocyte differentiation factor.

\section{Neuron-other glial cell interactions}

\section{Neuron-radial glia interactions}

During development of the CNS, neurons are born on the ventricular surface of the neural tube and appear to migrate to their final destination. The success of such event is partially dependent on the interaction of postmitotic neurons and the system of radial glial fibers, precursors of CNS astrocytes (52). Although this interaction is mainly mediated by contact and several proteins involved in this process have already been identified (4,53), there are few examples of soluble factors as mediators. Hunter and Hatten (54) have shown that the expression of radial glial cell identity in mammalian forebrain is determined by the availability of diffusible inducing signals. Although the factor has not been completely characterized, biochemical studies have indicated that it is different from the neural growth regulators already known. Those data provide support for the role of neuronal extrinsic signals in 
determining and maintaining a radial glial identity and suggest that transformation of radial glia into astrocytes is regulated by the availability of neuronal signals rather than by changes in cell potential (54). A remarkable example is the presence of the glutamate receptor GLAST in radial glia in the developing spinal cord (55). It is clear that glutamate transporters on mature astrocytes play an essential role in the rapid removal of extracellular glutamate in order to keep the level low enough to prevent neuronal excitotoxicity. Although their role in radial glia is not yet completely understood, it has been suggested that migration may be modulated by glutamate levels along the route. Furthermore, it is also important to consider that glutamate supplied by migrating neurons might also be involved in differentiation of the radial glia-astrocyte lineage.

GGF/neuregulin signaling has also been implicated in neuron-radial glial fiber interactions. Rakic's group (4) has shown that GGF is expressed by migrating cortical neurons and promotes their migration along radial glial fibers. Concurrently, GGF also promotes the maintenance and elongation of radial glial cells. In the absence of GGF signaling via erbB2 receptors radial glial development is abnormal. The ability of GGF to influence both neuronal migration and radial glial development in a mutually dependent manner suggests that it functions as a soluble mediator of migrating neurons and radial glial cells in the developing cerebral cortex.

\section{Neuron-Müller glia interactions}

The concept of glial-neuronal cell interactions in the retina during development has been extensively proposed. Fetal calf serum stimulates the proliferation of Müller cells, which indirectly arrests rod differentiation by releasing leukemia inhibitory factor (56). In addition, Wexler et al. (57) recently proposed a similar scheme in which brain-de- rived neurotrophic factor indirectly increased retinal bipolar cell survival in vitro by acting through p $75^{\text {NTR }}$ in Müller cells. The communication between Müller cells and retinal neurons indicates that these glial cells play an active role in retinal function. Recently, Harada et al. (58) have shown that Müller cells can prevent photoreceptor degeneration indirectly through bFGF production. Retinal degeneration up-regulated both p $75^{\text {NTR }}$ receptor and TrkC in different parts of Müller cells. Exogenous neurotrophin-3 increased but nerve growth factor decreased bFGF production in Müller cells which can directly rescue photoreceptor apoptosis. Although bFGF is known to stimulate rat photoreceptor survival directly through FGF receptors, the cited study demonstrated that cell survival during retinal degeneration may also be regulated in an indirect manner by glial cells and suggests functional glial-neuronal cell interactions as new therapeutic targets against neurodegeneration.

\section{Concluding remarks}

Taken together, available literature data support the concept that within the context of brain development, neuron-glia interactions are not of a single type, but rather present great complexity and heterogeneity throughout the CNS. Currently, it is widely accepted that neuron-glia interactions play an important role in several steps of nervous system morphogenesis from the early stages of neurogenesis and gliogenesis to later stages of establishment of neural connections. A dynamic interplay between neurons and glial cells undoubtedly helps to shape developing neural circuits by controlling the survival and morphology of neurons, the growth of their axons, and the number and efficacy of their synapses, as reported here based on data from different groups including ours. Although we have learned much about the physiology of glial cells over the last ten years, our knowledge of the function and 


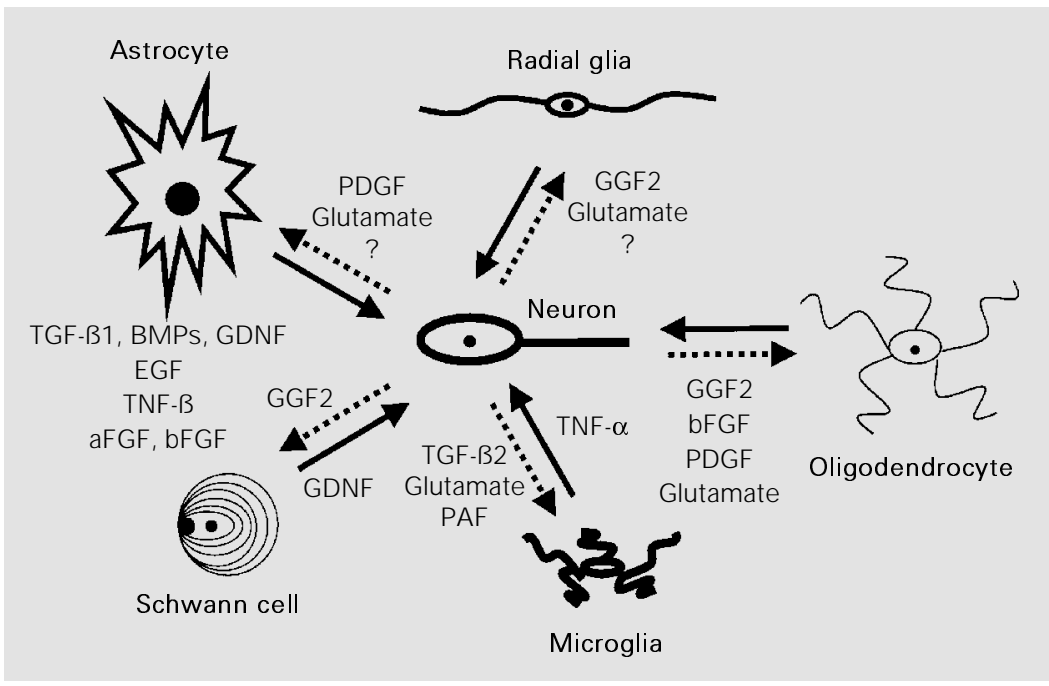

Figure 1. Neuron-glia interactions mediated by soluble factors. Secretion of growth factors by neurons and glial cells can mutually influence several steps of neurogenesis and gliogenesis (for details, see text). TGF-ß1 and TGF- $\$ 2$, transforming growth factor $ß 1$ and 2; TNF- $\alpha$ and TNF- $\beta$, tumor necrosis factor alpha and beta; PAF, platelet-activating factor; EGF, epidermal growth factor; PDGF, platelet-derived growth factor; bFGF and aFGF, basic and acidic fibroblast growth factor; BMPs, bone morphogenetic protein family; GGF2, glial growth factor 2; GDNF, glial cell line-derived neurotrophic factor.

development of glia is still rudimentary. Several growth factors involved in gliogenesis have been identified and this will certainly be crucial for a better understanding of glial cell functions and interactions with neurons. The recent finding that subventricular astrocytes can act as neural stem cells in the adult mammalian brain clearly highlights the new view of glial cells held by neurobiologists (59). Whereas glial cells have been regarded so far as elements of structural and trophic support, today they might represent a key element in neural cell origin and therefore in brain development.

One key issue in developmental neurobiology is to understand how the brain orchestrates the differentiation of various cell types. A range of epigenetic signals are involved in neural fate potential, lineage specification and cellular differentiation in the CNS and peripheral nervous system. Some of these signals are initiated very early in development due to the diffusible factors and cell contact found in the developmental environment.
Within this context, it will be very useful in the future to elucidate the mechanisms involved in neuron-glia interactions, since these are among the most relevant interactions neural cells will experience during development. Although several molecules involved in such interactions have already been identified (Figure 1), we are still in the dark concerning neuronal effects on glial cells particularly those involved in astrocyte development. It would be worth to explore gene expression in neural cells in order to understand how neuron-glia interactions might modulate developmental genes during construction of the nervous system.

One of the most compelling lines of evidence for the role of neuron-glia interactions in nervous system development was the identification of the glial cell missing $(\mathrm{gcm})$ gene in Drosophila (60) which functions as a binary switch that turns on glial fate while inhibiting default neuronal fate. Its mutation causes presumptive glial cells to differentiate into neurons, whereas its ectopic expression forces virtually all CNS cells to become glial cells. Analysis of $\mathrm{gcm}$ mutants revealed, in addition to a decreased number of glial cells, a series of defects in several axonal tracts. Such defects were attributed mainly to the loss of glial signals important to axonal growth and neuronal proliferation and differentiation (60).

Until recently there was no way to selectively eliminate mammalian glial cells in vivo in order to explore how the brain develops and functions without them, as done in Drosophila after $\mathrm{gcm}$ identification. However, we have taken a great step from the passive glia described by Virchow nearly a century ago to the astrocyte stem cells of today. The close association between neurons and glial cells during nervous system development suggests that deep inside these interactions might be hidden the secrete of the nervous system organization. 


\section{References}

1. Lim DA \& Alvarez-Buylla A (1999). Interaction between astrocytes and adult subventricular zone precursors stimulates neurogenesis. Proceedings of the $\mathrm{Na}$ tional Academy of Sciences, USA, 96: 7526-7531.

2. Barres BA (1997). Neuron-glial interactions. In: Cowan WM, Jessell TM \& Zpursky SL (Editors), Molecular and Cellular Approaches to Neural Development. Oxford University Press, New York, 64107.

3. Pfrieger FW \& Barres BA (1997). Synaptic efficacy enhanced by glial cells in vitro. Science, 277: 1684-1686.

4. Anton ES, Marchionni MA, Lee KF \& Rakic $P$ (1997). Role of GGF/neuregulin signaling in interactions between migrating neurons and radial glia in the developing cerebral cortex. Development, 124: 35013510.

5. Gomes FCA, Maia CG, Menezes J RL \& Moura Neto V (1999). Cerebellar astrocytes treated by thyroid hormone induce neuronal proliferation. Glia, 25: 247-255.

6. Garcia-Abreu J, Moura Neto V, Carvalho SL \& Cavalcante LA (1995). Regionally specific properties of midbrain glia: I. Interactions with midbrain neurons. J ournal of Neuroscience Research, 40: 417-477.

7. Fróes MM, Correia AHP, Garcia-Abreu J , Spray DC, Campos de Carvalho AC \& Moura Neto V (1999). J unctional coupling between neurons and astrocytes in primary CNS cultures. Proceedings of the National Academy of Sciences, USA, 96: 7541-7546.

8. Alvarez-Maubecin V, García-Hernández F, Williams J T \& Van Bockstaele EJ (2000). Functional coupling between neurons and glia. J ournal of Neuroscience, 20: 40914098.

9. Giulian D, Allen RL, Baker TJ \& Tomozawa $Y$ (1986). Brain peptides and glial growth. I. Glia-promoting factors as regulators of gliogenesis in the developing and injured central nervous system. J ournal of Cell Biology, 102: 803-811.

10. David S, Miller RH, Patel R \& Raff MC (1984). Effects of neonatal transection on glial cell development in the rat optic nerve: evidence that the oligodendrocytetype-2 astrocyte cell lineage depends on axons for its survival. J ournal of Neurocytology, 13: 961-974.

11. Kidd GJ, Hauer PE \& Trapp BD (1990). Axons modulate myelin protein messenger RNA levels during central nervous sys- tem myelination in vivo. J ournal of Neuroscience Research, 26: 409-418.

12. Asakura $K$, Hunter SF \& Rodriguez $M$ (1997). Effects of transforming growth factor- $\beta$ and platelet-derived growth factor on oligodendrocyte precursors: insights gained from a neuronal cell line. J ournal of Neurochemistry, 68: 22812290.

13. Wood PM \& Bunge RP (1986). Evidence that axons are mitogenic for oligodendrocytes isolated from adult animals. Nature, 320: 756-758.

14. Noble $M$, Murray $K$, Stroobant $P$, Waterfield MD \& Riddle P (1988). Platelet-derived growth factor promotes division and motility and inhibits premature differentiation of the oligodendrocyte/type-2 astrocyte progenitor cell. Nature, 333: 560562.

15. Grinspan J B, Edell E, Carpio DF, Beesley J S, Lavy L, Pleasure D \& Golden J A (2000). Stage-specific effects of bone morphogenetic proteins on the oligodendrocyte lineage. J ournal of Neurobiology, 43: 1-17.

16. Canoll PD, Musacchio JM, Hardy R, Reynolds R, Marchionni MA \& Salzer J L (1996). GGF/neuregulin is a neuronal signal that promotes the proliferation and survival and inhibits the differentiation of oligodendrocyte progenitors. Neuron, 17: 229-243.

17. Dong Z, Brennan A, Liu N, Yarden $Y$, Lefkowitz G, Mirsky $R$ \& J essen KR (1995). Neu differentiation factor is a neuron-glia signal and regulates survival, proliferation, and maturation of rat Schwann cell precursors. Neuron, 15: 585-596.

18. Maurel P \& Salzer J L (2000). Axonal regulation of Schwann cell proliferation and survival and the initial events of myelination requires $\mathrm{PI}$ 3-kinase activity. J ournal of Neuroscience, 20: 4635-4645.

19. Syroid DE, Maycox PR, Burrola PG, Liu N, Wen $D$, Lee $K F$, Limke $G$ \& Kilpatrik TJ (1996). Cell death in the Schwann cell lineage and its regulation by neuregulin. Proceedings of the National Academy Sciences, USA, 93: 9229-9234.

20. Meyer D \& Birchmeier C (1995). Multiple essential functions of neuregulin in development. Nature, 278: 386-390.

21. Shah NM, Marchionni MA, Issacs I, Stoobant P \& Anderson DJ (1994). Glial growth factor restricts mammalian neural crest stem cells to a glial fate. Cell, 77 : 349-360.
22. Cameron HA, Hazel TG \& McKay RDG (1998). Regulation of neurogenesis by growth factors and neurotransmitters. J ournal of Neurobiology, 36: 287-306.

23. Gallo V, Zhou J M, McBain CJ , Wright PW Knutson PL \& Armstrong RC (1996). Oligodendrocyte progenitor cell proliferation and lineage progression are regulated by glutamate receptor mediated $\mathrm{K}^{+}$channel block. J ournal of Neuroscience, 16: 26592670

24. Yuan X, Eisen AM, McBain CJ\& Gallo V (1998). A role for glutamate and its receptors in the regulation of oligodendrocyte development in cerebellar tissue slices. Development, 125: 2901-2914.

25. Ling EA \& Wong WC (1993). The origin and nature of ramified and amoeboid microglia: a historical review and current concepts. Glia, 7: 9-18.

26. Dobbertin A, Schmid P, Gelman M, Glowinski J \& Mallat M (1997). Neurons promote macrophage proliferation by producing transforming growth factor-ß2. J ournal of Neuroscience, 17: 5305-5315.

27. Derynck R \& Feng X-H (1997). TGF-ß receptor signaling. Biochimica et Biophysica Acta, 1333: F105-F150.

28. Noda M, Nakanishi H, Nabekura J \& Akaike N (2000). AMPA-kainate subtypes of glutamate receptor in rat cerebral microglia. J ournal of Neuroscience, 20: 251258.

29. Aihara $M$, Ishii $S$, Kume $K \&$ Shimizu $T$ (2000). Interaction between neurone and microglia mediated by platelet-activating factor. Genes to Cells, 5: 397-406.

30. Connor B \& Dragunow M (1998). The role of neuronal growth factors in neurodegenerative disorders of the human brain. Brain Research Reviews, 27: 1-39.

31. Banker GA (1980). Trophic interactions between astroglial cells and hippocampal neurons in culture. Science, 209: 809-810.

32. Kane CJ M, Brown GJ \& Phelan KD (1996). Transforming growth factor-ß2 stimulates and inhibits neurogenesis of rat cerebellar granule cells in culture. Developmental Brain Research, 96: 46-51.

33. Kuhn HG, Winkler J , Kempermann G, Thal LJ \& Gage FH (1997). Epidermal growth factor and fibroblast growth factor-2 have different effects on neural progenitors in the adult rat brain. J ournal of Neuroscience, 17: 5820-5829.

34. Bruno V, Battaglia G, Casabona G, Copani A, Caciagli F \& Nicoletti F (1998). Neuroprotection by glial metabotropic glutamate 
receptors is mediated by transforming growth factor-ß3. J ournal of Neuroscience, 18: 9594-9600.

35. Oppenheim RW, Houenou LJ, Parsadanian AS, Prevette $D$, Snider WD \& Shen L (2000). Glial cell line-derived neurotrophic factor and developing mammalian motoneurons: regulation of programmed cell death among motoneuron subtypes. J ournal of Neuroscience, 20: 5001-5011.

36. Gomes FCA, Lima FRS, Trentin AG, Mallat M \& Moura Neto V (2000). Thyroid hormone role on nervous system morphogenesis. Progress in Brain Research (in press).

37. Stoeckli A \& Landmesser LT (1998). Axon guidance at choice points. Current Opinion in Neurobiology, 8: 73-79.

38. Serafini $T$, Colamarino $S A$, Leonardo ED, Wang $\mathrm{H}$, Beddington $\mathrm{R}$, Skarnes WC \& Tessier-Lavigne M (1996). Netrin-1 is required for commissural axon-guidance in the developing vertebrate nervous system. Cell, 87: 1001-1014.

39. ProchiantzA (1995). Neuronal polarity: giving neurons heads and tails. Neuron, 15: 743-746.

40. Garcia-Abreu J, Mendes FA, Onofre GR, Freitas MS, Silva LCF, Moura Neto V \& Cavalcante $L$ (2000). Contribution of heparan sulfate to the non-permissive role of the midline glia to the growth of midbrain neurites. Glia, 29: 260-272.

41. Karshing $A$, Wassle $H \&$ Schnitzer J (1986). Shape and distribution of astrocytes in the cat retina. Investigative Ophthalmology and Visual Science, 26: 828831.

42. Fruttiger $M$, Calver $A R$, Krüger $W H$, Mudhar HS, Michalovich D, Takakura N, Nishikawa SI \& Richardson WD (1996). PDGF mediates a neuron-astrocyte interaction in the developing retina. Neuron, 17: 1117-1131.

43. Gargini C, Deplano S, Bisti S \& Stone J (1998). Evidence that the influence of ganglion cell axons on astrocyte morphology is mediated by action spike activity during development. Developmental Brain Research, 110: 177-184.

44. Swanson RA, Liu J, Miller J W, Rothstein J D, Farrel K, Stein BA \& Longuemare MC (1997). Neuronal regulation of glutamate transporter subtype expression in astrocytes. J ournal of Neuroscience, 17: 932940.

45. Hatten ME (1985). Neuronal regulation of astroglia morphology and proliferation in vitro. J ournal of Cell Biology, 100: 384396.

46. Gomes FCA, Paulin D \& Moura Neto V (1999). GFAP: modulation by growth factors and its implication in astrocyte differentiation. Brazilian J ournal of Medical and Biological Research, 32: 615-631.

47. Gomes FCA, Garcia-Abreu J, Galou M, Paulin D \& M oura Neto V (1999). Neurons induce glial fibrillary acidic protein (GFAP) gene promoter of astrocytes derived from transgenic mice. Glia, 26: 97-108.

48. Kvamme E, Svenneby G, Hertz L \& Schousboe A (1982). Properties of phosphate activated glutaminase in astrocytes cultured from mouse brain. Neurochemical Research, 7: 761-770.

49. Würdig $S \&$ Kugler $P(1990)$. Histochemistry of glutamate metabolising enzymes in the rat cerebellar cortex. Neuroscience Letters, 130: 165-168.

50. Gegelashvili G, Dehnes Y, Danbolt NC \& Schousboe A (2000). The high-affinity glutamate transporters GLT1, GLAST, and EAAT4 are regulated via different signaling mechanisms. Neurochemistry International, 37: 163-170.

51. Perego C, Vanoni C, Bossi M, Massari S, Basudev H, Longhi R \& Pietrini G (2000). The GLT-1 and GLAST glutamate transporters are expressed on morphologically distinct astrocytes and regulated by neuronal activity in primary hippocampal cocultures. J ournal of Neurochemistry, 75: 1076-1084.

52. Pearlman $A L$, Faust $P L$, Hatten ME \&
Brunstrom J E (1998). New directions for neuronal migration. Current Opinion in Neurobiology, 8: 45-54.

53. Zhen L, Heintz N \& Hatten ME (1996). CNS gene encoding astrotactin, which supports neuronal migration along glial fibers. Science, 272: 417-419.

54. Hunter KE \& Hatten ME (1995). Radial glial cell transformation to astrocytes is bidirectional: Regulation by a diffusible factor in embryonic forebrain. Proceedings of the National Academy of Sciences, USA, 92: 2061-2065.

55. Shibata $T$, Yamada $K$, Watanabe $M$, Ikenaka K, Wada K, Tanaka K \& Inoue Y (1997). Glutamate transporter GLAST is expressed in the radial glia-astrocyte lineage of developing mouse spinal cord. J ournal of Neuroscience, 17: 9212-9219.

56. Neophytou $C$, Vernallis $A B$, Smith $A \&$ Raff MC (1997). Müller-cell-derived leukaemia inhibitory factor arrests rod photoreceptor differentiation at postmitotic prerod stage of development. Development, 124: 2345-2354.

57. Wexler EM, Berkovich $O \&$ Nawy $S$ (1998). Role of low-affinity NGF receptor (p75) in survival of retinal bipolar cells. Visual Neuroscience, 15: 211-218.

58. Harada T, Harada C, Nakayama N, Okuyama S, Yoshida K, Kohsaka S, Matsuda H \& Wada K (2000). Modification of glialneuronal cell interactions prevents photoreceptor apoptosis during light-induced retinal degeneration. Neuron, 26: 533541.

59. Doetsch F, Caillé I, Lim DA, GarcíaVerdugo J M \& Alvarez-Buylla A (1999). Subventricular zone astrocytes are neural stem cells in the adult mammalian brain. Cell, 97: 703-716.

60. J ones BW, Fetter RD, Tear G \& Goodman CS (1995). Glial cells missing: a genetic switch that controls glial versus neuronal fate. Cell, 82: 1013-1023. 